\title{
Time Scheduling Schemes in Online Laboratory Management Systems
}

\author{
doi:10.3991/ijoe.v6i4.1361 \\ Ananda Maiti ${ }^{1}$ \\ ${ }^{1}$ Indian Institute of Technology, Kharagpur, India
}

\begin{abstract}
Online remotely-controlled educational laboratories are increasingly being deployed in many universities around the world. As hardware-based remote experiment can only be used by one person or a group of persons at a time, for proper utilization of the resources, an efficient time scheduling scheme is essential. In this work, we discuss some of the possible methods for scheduling of online experiments which may be integrated in online laboratory management systems for optimized resource utilization.
\end{abstract}

Index Terms — online laboratory, time-sharing, queuing, scheduling

\section{INTRODUCTION}

The last five decades have seen steady advancements in the field of microelectronics and thus the training of electronic engineers in microelectronics area has become imperative. It is also universally accepted that hands-on laboratory experience is essential for mastery of the fundamentals of microelectronics. However, as the semiconductor measurement and characterization equipment and the test units are generally very expensive, creation of microelectronics laboratory has been very limited. Very few institutions can even afford an undergraduate microelectronics laboratory due to the high start-up costs, high equipment cost and regular maintenance of such equipment. As the broadband connectivity to the Internet is becoming very common, development of web-based remotely controlled semiconductor devices laboratory has come into play an important role in microelectronics laboratory education, where the learners have much more flexibility in performing the experiments at any time and from anywhere [1,2].

A new undergraduate microelectronic devices and VLSI Engineering laboratory developed at IIT Kharagpur is interactive in nature and gives the students a realistic exposure to the semiconductor device characterization and other experiments. The student must first schedule a time to use the laboratory. At the scheduled time, the student logs in and is able to launch the laboratory client. As the experiment requires real-time control of the equipment, the laboratory currently uses time-slot during which the students set the measurement conditions and conduct experiments from the remote PC. However, the students can read manuals; analyze the measured data without occupying the measuring equipments.

Interactive experiments require the control of instruments during which the users set the parameters and observe the results and are fundamentally different from the batch-mode (queue) counterparts. Even so, it is difficult to provide the equipments to students for long periods of time as the actual measurement time is usually very short. Most of the time is spent for measurement condition setting up and post experiment data analysis. Thus, for proper utilization of the resources, a proper scheduling is necessary in the laboratory management system. In order to choose the service provider with the different kinds of measurement equipments and the target devices, in this paper, we present the results of our study on scheduling experiments to achieve an efficient equipment-sharing in case of multiple student requests arriving at the same time.

\section{SCHEDULING SCHEMES}

As discussed above, some of the possible scheduling schemes are:

i. Time Slot mode

ii. Queuing mode

iii. Slotted-queuing mode

\section{A. Time Slot Mode}

When a student prepares to conduct an experiment involving a unique experimental setup (i.e., one that cannot be duplicated for concurrent use by others), one need to find an available time slot and then register for the slot (Fig. 1). The time slot (duration) is the time necessary to carry out the experiment and needs to be pre-estimated. The scheduling system for online experiment described here also assumes that the student would be able to finish the experiments during this time, just like they would do in a conventional laboratory.

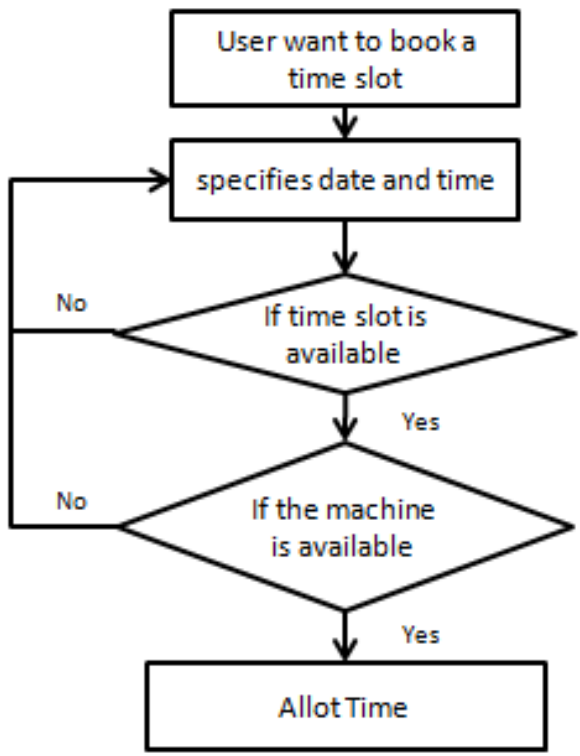

Figure 1. Flowchart of Time Slot Method 


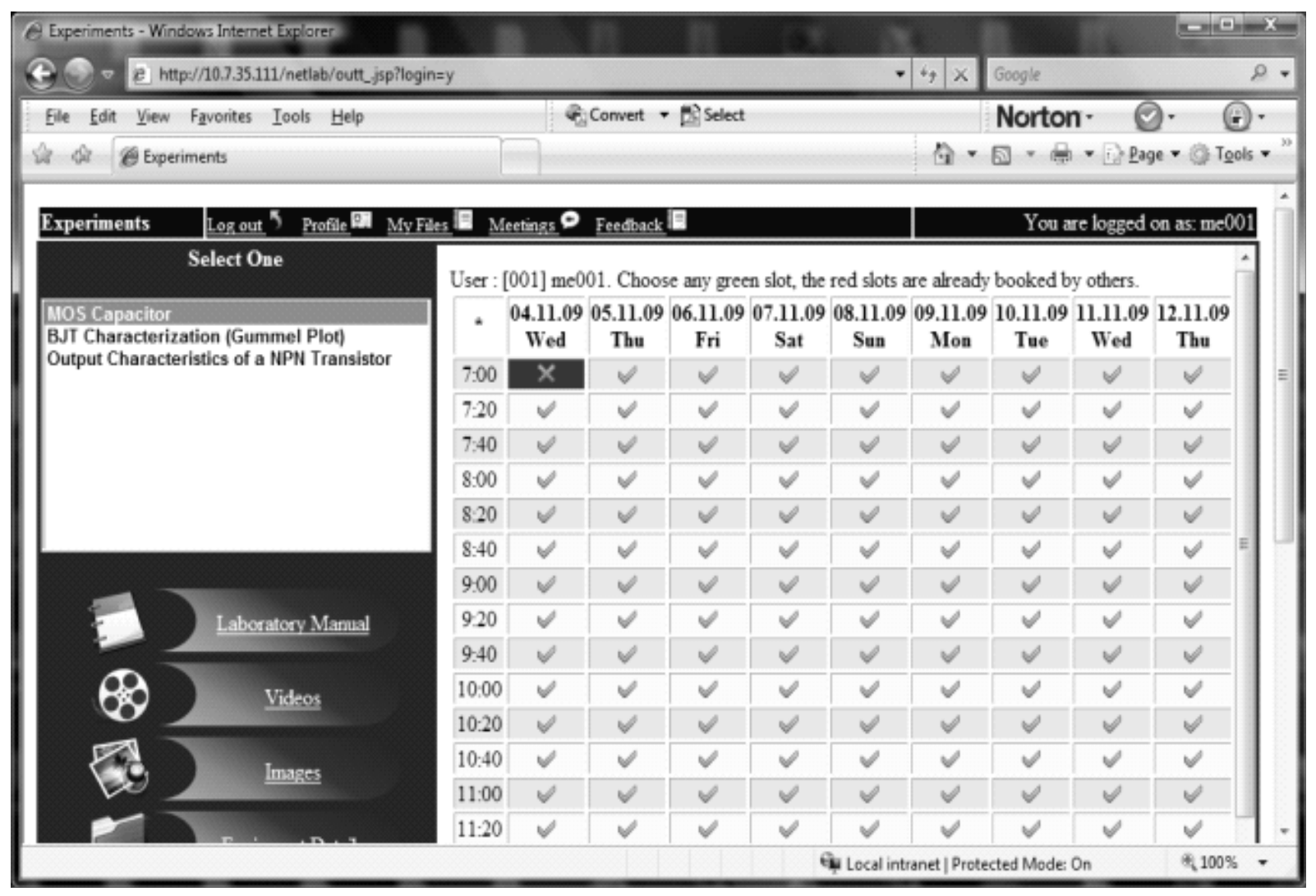

Figure 2.

Each experiment is associated with a given time length which the administrator thinks is enough for the experiment, keeping in view the speed of Internet and other network elements.

The workflow may be summarized as follows:

- Selection of the experiment

- Opening of the scheduling web page link in the experiment page

- The system displays the scheduling interface for the next 15 days

- The system displays the available time slots for the selected experiment in green color

- The learner selects the date/time for carrying out the experiment in the scheduling interface

- The learner selects a desired time slot from the available time slots and submits the request

- The system saves the learner schedule information and updates the scheduling database.

The system displays a confirmation of the schedule to the learner. In due course the user is given a temporary link to the web page through which the equipment can be accessed and be controlled. After the specified amount of time a JavaScript automatically closes the window with a warning.

The followings are some of the advantages:

i. The user gets the full control of the equipment/system and performs the experiment at any time he/she wants. The users do not have to wait.

ii. Ideally suited for experiments where several measurements are necessary under different measuring conditions.
However, main disadvantages are:

i. The utilization of the resources is poor and the efficiency of the management system is not high since the equipment is not always in use and is sitting idle for some time

ii. Number of users accessing the resources is very low and not satisfactory.

\section{B. Queuing}

The usefulness and efficiency of online laboratories may be improved, if several users could use the system in a short period of time and even concurrently. In this method, the users set up measurement parameters and issue the request to perform the experiment, regardless of what others are doing (Figure 3). The users need not get a particular slot of time of their own. However, a user might have to wait for some time before getting the data.

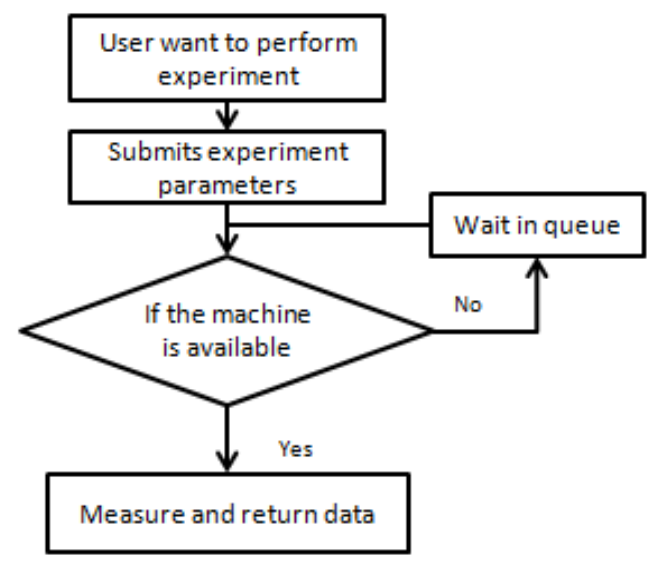

Figure 3. Flowchart of Queuing Method 


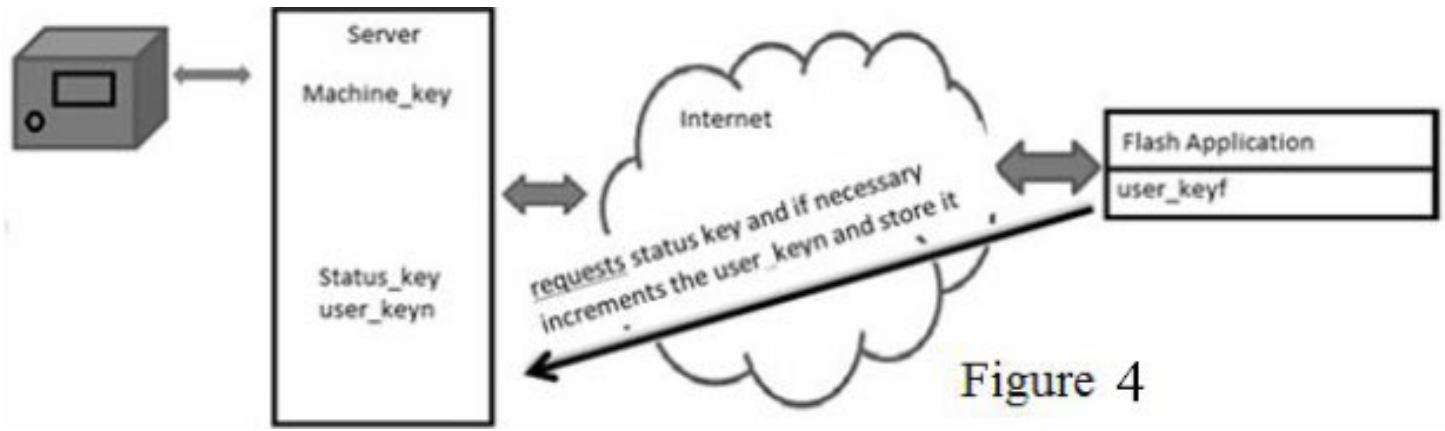

Figure 4.

In this case, a status key and the user key in the database of the server controlling the equipments are used to keep track of the device status. If the device is in use then the status key is set to 1 , otherwise 0 . The user key holds the number of users waiting in queue at any point of time. The front end of the system is a web browser, containing a flash application.

This flash application uses a web service to run the equipment. Before it runs this web method, it runs another web method to retrieve the value of the keys of the respective machine. If the value of the status key is 0 , the current value of the user key is stored in the flash application and the user key is increased by 1 and then the application sleeps for 1 second (typically) and the process continues again. If the value of the status key is found to be 1 and the value of the user key is 0 , then the next web method is called, otherwise, the application sleeps for 1 second and the process continues again (Figure 4).

Web services are Application Programming Interfaces (API) or web APIs that can be accessed over a network, such as the Internet, and may be executed on a remote system hosting the requested services.

Basically, if one user is using the equipment (performing the measurement) then other users wait in the queue. When the current user's request is finished then the next user's request in the queue is processed and so on. If any of the users becomes unavailable during the waiting time, the system waits for that user's request for some time and then takes up the next.

Some of the advantages are:

i. The efficiency of the management system is optimal since the machine is always in use and is not sitting idle.

ii. Ideally suited for experiments with less number of measurements i.e., the number of times the equipment is measuring is higher than the number of times the measurement conditions are changed.

The disadvantages are:

i. A user does not get full control of the system/equipment and cannot run it at any time he/she wants.

ii. The user has to wait for some time and waiting may be large (depending on the number of users in the queue).

\section{Slotted Queuing}

Both the above methods have their own problems that make them unsuitable for most experiment. Probably, the best way to overcome the shortcomings for the methods viz., the time-slot and queuing methods (as discussed above) is to combine the two. In this approach, in a timeslot, more than one user is allowed to book the time. However, the number is fixed and is decided by the administrator or the tool developer. All the user booked in that particular time slot follow the simple queuing method. When the devices are engages in an experiment by a user other will have to wait for a certain time (Figure 5). Let's assume that the number of allowed users be $\mathrm{n}$ in a particular time slot. Then in the worst case scenario, the maximum waiting time (Wtmax) is given by:

$$
W_{\text {tmax }}=(n-1) \times t
$$

where $t$ is the time taken to perform the experiment.

The number of chances, one user gets is given by

$$
N c=\frac{T}{n \times t}
$$

where $\mathrm{T}$ is the duration of the time slot and may also be expressed as:

\section{Nc $\propto T$}

Thus, the developer has to maintain a balance between the number of chances a user needs to have to complete the experiment and the total duration of the time slot. Thus,

$$
T=\left(W_{t \max }+t\right) \times N c
$$

and

$$
n=\frac{W_{t \max }}{t}+1
$$

From the above, one needs to determine the number of chances required to complete the experiment and the maximum waiting time allowed according to the resources available in the laboratory. Once this is fixed, one can find out what should be the suitable duration of time slot and the number of users that can be allowed to book their time in the slot.

The advantages of this method are:

i. It ensures that the queue will not be very long and also the waiting time. This will help one to have access to the laboratory at any point of time.

ii. Suitable for any type of experiment.

iii. The efficiency of the management system is better than time slot and hence the resource utilization. 


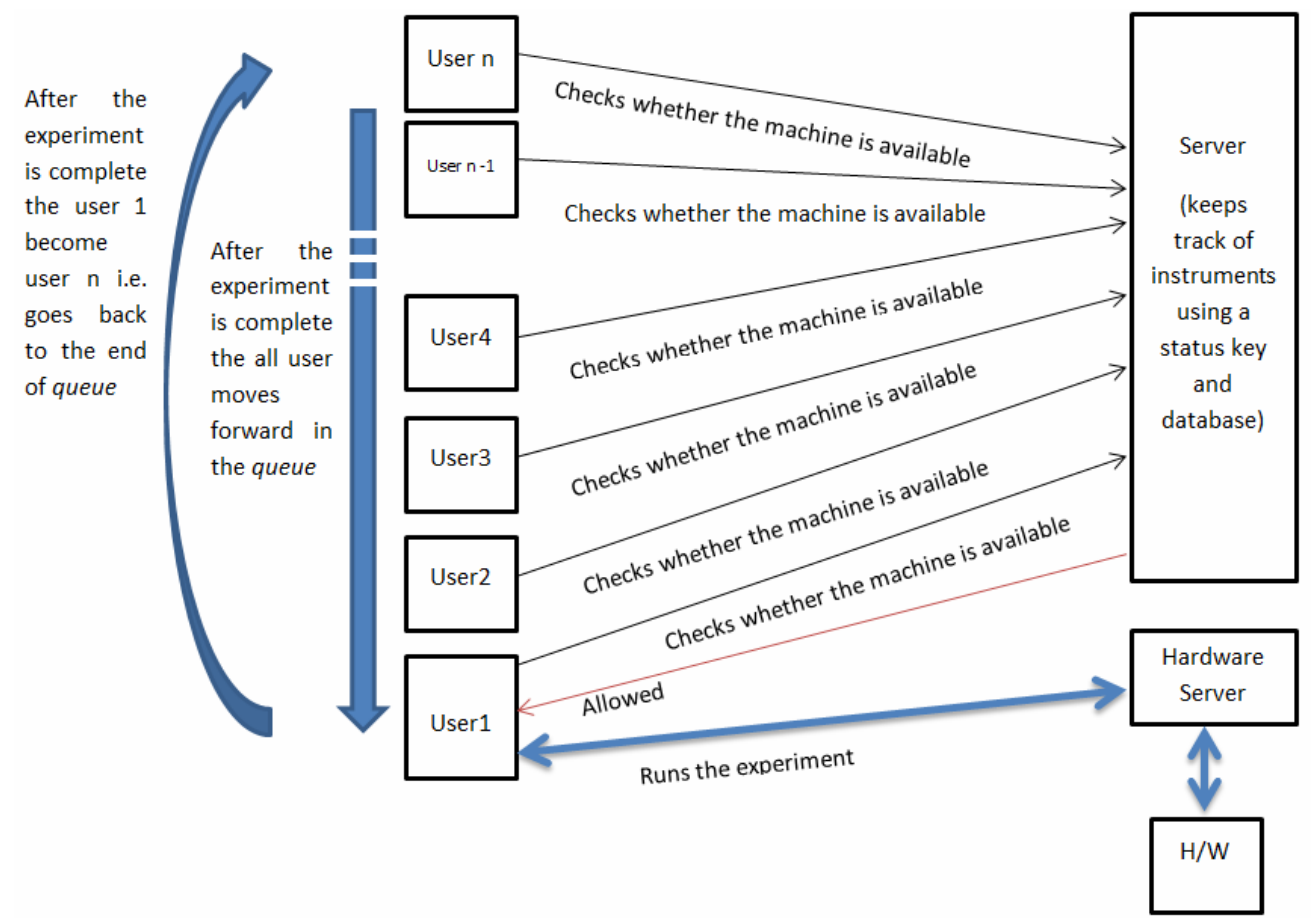

Figure 5. The slotted queuing mode (here $\mathrm{n}$ is fixed number)

\begin{tabular}{|c|c|c|c|c|}
\hline E1 & DAY1 & DAY2 & DAY3 & DAY4 \\
\hline T1 & $n=1$ & $n=1$ & $n=2$ & $n=1$ \\
\hline T2 & $n=1$ & $n=2$ & $n=1$ & $n=2$ \\
\hline T3 & $n=1$ & $n=1$ & $n=1$ & $n=2$ \\
\hline T4 & $n=2$ & $n=1$ & $n=0$ & $n=2$ \\
\hline
\end{tabular}

Figure 6(a)

It is obvious that if the number of the users is more in each slot, the efficiency of the system will be higher and will reach that of queuing method. However, if the users book their time individually in separate slots and thus lowering the number of users per slot, the efficiency goes down and will close to time slot method as is illustrated below for an experiment (E1).

In the 1st case the number of user is higher (Figure 6a) and closer to 5 (assumed to be the maximum number of user allowed in each slot). In the above analysis, it is assumed that the available experiment is only one. Thus the efficiency is more than in the 2nd case (Figure 6b) where the situation is almost the same as that of time slot method. This scenario is expected, if all the users want to do different experiments on the same day and the same device is used to perform different experiments (E1, E2 ...).

However, if a user is permitted to perform more than one experiment during the same time slot $(\mathrm{Ti})$ then the number users can be made higher for an experiment (Ei). The other way to improve the efficiency is to allow each user to book more than one time slot and the user will get more number of chances. Thus, the duration of the time slots can be further reduced.

The slotted-queuing method is currently being used successfully in NetLAB laboratory management tool at

\begin{tabular}{|c|c|c|c|c|}
\hline E1 & DAY1 & DAY2 & DAY3 & DAY4 \\
\hline T1 & $n=1$ & $n=1$ & $n=2$ & $n=1$ \\
\hline T2 & $n=1$ & $n=2$ & $n=1$ & $n=2$ \\
\hline T3 & $n=1$ & $n=1$ & $n=1$ & $n=2$ \\
\hline T4 & $n=2$ & $n=1$ & $n=0$ & $n=2$ \\
\hline
\end{tabular}

Figure 6(b)

IIT Kharagpur [3]. In Figures 7 and 8, we show that more than one user can book time in one slot (as compared to Figure 2) and can perform the same experiment or different experiments can be performed by the same user. A user may see the Number of Users/Maximum users allowed in a slot during the time slot booking (see Figure 7).

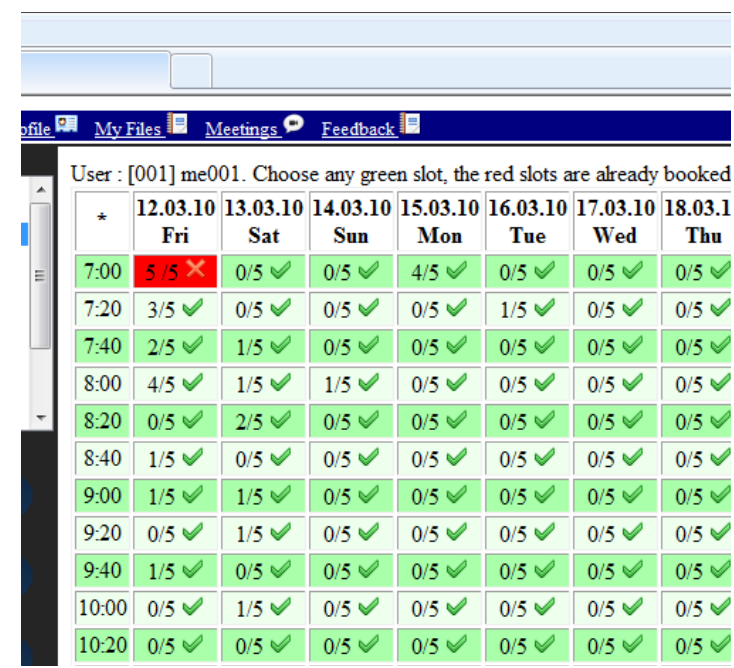

Figure 7. Time Booking. 
Group : 1

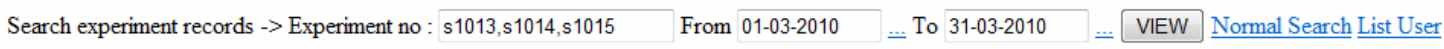

\begin{tabular}{|c|c|c|c|c|c|}
\hline expno & exp name & time & u code & u name & A \\
\hline s1014 & Collector Resistance & $18-03-10 \quad 19: 00$ & 08EC1003(00092) & Hiranmoy Basak & \\
\hline s1014 & Collector Resistance & $18-03-10$ 19:00 & $08 \mathrm{ec} 3005(00229)$ & mayank mundra & \\
\hline s1015 & HFE Charateristics & $18-03-1020: 00$ & $08 \mathrm{ec} 3005(00229)$ & mayank mundra & \\
\hline s1015 & HFE Charateristics & $18-03-1020: 00$ & 08EC1005(00103) & Naribole Sharan & \\
\hline s1015 & HFE Charateristics & $18-03-1018: 15$ & 08EC3028(00208) & mahesh kumar & \\
\hline s1015 & HFE Charateristics & $18-03-1018: 15$ & 08EC3026(00202) & Debnath Maji & \\
\hline s1015 & HFE Charateristics & $18-03-10$ 19:15 & 08EC1003(00092) & Hiranmoy Basak & \\
\hline s1013 & Emitter resistance & $18-03-1018: 00$ & 08EC1006(00170) & Harsh Gupta & \\
\hline s1015 & HFE Charateristics & $18-03-1018: 45$ & $08 \mathrm{ec} 3027(00191)$ & Amarkant Kumar & \\
\hline s1015 & HFE Charateristics & $18-03-10$ 19:00 & $08 \mathrm{ec} 1043(00228)$ & Tapas Khanda & \\
\hline s1014 & Collector Resistance & $18-03-10 \quad 19: 15$ & $08 \mathrm{ec} 1041(00096)$ & atin mondal & \\
\hline s1013 & Emitter resistance & $18-03-10 \quad 19: 45$ & $08 \mathrm{ec} 1043(00228)$ & Tapas Khanda & \\
\hline s1013 & Emitter resistance & $18-03-1020: 00$ & 08EC3025(00157) & Swagata Biswas & \\
\hline s1013 & Emitter resistance & $18-03-1020: 15$ & 08EC3025(00157) & Swagata Biswas & \\
\hline s1014 & Collector Resistance & $18-03-1020: 15$ & 08EC3025(00157) & Swagata Biswas & \\
\hline s1013 & Emitter resistance & $18-03-1020: 30$ & 08EC1030(00233) & Naveen Kumar Bansal Bansal & E \\
\hline s1014 & Collector Resistance & $18-03-1020: 30$ & 08EC1030(00233) & Naveen Kumar Bansal Bansal & - \\
\hline
\end{tabular}

Figure 8. This is an excerpt from the logbook of NetLAB showing the users and the time slot in which they have worked. The same experiment is being done by more than one user (black enclosure) and different experiments are being done by different users (red enclosure) in one time slot.

\section{CONCLUSION}

For an interactive laboratory session, the laboratory hardware needs to be dedicated to a single user for the duration of the experiment depending on the nature of the experiment and hence require a proper scheduling scheme. Existing remote laboratories limit the number of users to the number of actual available resources. By integrating mixed-mode scheduling services in a laboratory management system, it is shown that a diverse set of experiments can easily be deployed online and resources shared and thus optimizing the resource utilization.

\section{REFERENCES}

[1] J. L. Hardison, K. DeLong, P. H. Bailey, and V. J. Harward, "Deploying Interactive Remote Labs Using the iLab Shared Architecture”, FIE, pp. S2A-1 - S2A-6, 2008.
[2] A. Maiti, Invited, "Online Microelectronics and VLSI Engineering Laboratory Management System”, ICVLSICOM, Jan 10, 2010.

[3] A. Maiti, "NETLab: An Online Laboratory Management System”, iJOE, vol. 6, pp. 31-36, 2010.

\section{AUTHORS}

A. Maiti is with the Indian Institute of Technology, India and is involved with development of the Virtual Laboratories (e-mail: anandamaiti@live.com).

Submitted June $23^{\text {rd }}$, 2010. Published as resubmitted by the authors October $17^{\text {th }}, 2010$. 